\title{
AN INTELLIGENT VIRTUAL ENVIRONMENT FOR DISTANCE LEARNING
}

\author{
Cássia Trojahn dos Santos and Fernando Santos Osório \\ Unisinos University, Brazil
}

\begin{abstract}
This paper presents an intelligent, adaptive, three-dimensional and virtual environment for distance learning. The environment, used to make educational content available, has its structure and presentation customized according to users' interests and preferences (represented in user models) and in accordance with insertion or removal of contents. An automatic categorization process is applied in the creation of content models, used in the spatial organization of the contents in the environment. Moreover, the environment is populated by an intelligent agent, who assists users during the navigation and retrieval of relevant information.
\end{abstract}

Key words: intelligent virtual environments; virtual reality; distance learning.

\section{INTRODUCTION}

Virtual Reality (VR) became an attractive alternative for the development of more realistic and interesting three-dimensional visual interfaces for the user [Teichrieb, 1999]. The environments that make use of VR techniques are referred as Virtual Environments (VEs). In VEs, according to Avradinis et al. (2000), the user is a part of the system, an autonomous presence in the environment. He is able to navigate, to interact with objects and to examine the environment from different viewpoints. According to Frery et al. (2002), the 3D paradigm is useful mainly because it offers the possibility of representing information in a realistic way, while it organizes the contents in a spatial manner. In this way, a larger intuition in the visualization of the information is obtained, allowing the user to explore it in an interactive way, more natural to humans. Moreover, a more effective interaction can be 
obtained from the adaptation of interface, according to the characteristics of individual users or groups of users. According to Chittaro and Ranon (2002), the capability of (semi)automatically adapting the content, structure, and/or presentation of environment, to address the users' interests and preferences, is considered as a key factor to increase user satisfaction.

Moreover, nowadays, the focus of attention has been the integration of Artificial Intelligence (AI) and VEs. The objective is to obtain larger usability and realism from the interfaces, exploring the combination of 3D objects and intelligent entities. According to Aylett and Luck (2000), the environments that explore such integration are called Intelligent Virtual Environments (IVEs). An IVE is a virtual environment resembling the real world, populated by autonomous intelligent entities capable of a variety of behaviors. These entities may be simple, static or dynamic objects, virtual representations of life forms (virtual animals and humans), avatars of real world users entering the system, and others [Anastassakis et al. 2001]. According to Rickel et al. (2002), the potential applications for these environments are substantial, since they could be applied in a variety of areas, mainly related to simulation, entertainment and education.

In this paper, an intelligent and adaptive virtual environment for distance learning is presented. The paper is organized as follows. In section 2, the proposed environment is presented. Section 3 comments on the prototype developed to validate the environment. In section 4 , the final considerations are presented.

\section{THE INTELLIGENT VIRTUAL ENVIRONMENT}

The proposed environment consists of the representation of a 3D world, accessible through the Web, used to make educational content available. In this environment, there is support for two types of users: information consumer (e.g., student) and information provider (e.g., teacher). The consumer, represented by avatars, explores the environment searching relevant contents and can be aided by the virtual agent, in the navigation and location of information. A consumer model is maintained, so that the environment can be adapted according to this model. The providers, responsible for made available contents, can explore the environment. A provider model also is kept. The consumer and provider models are managed by the user model manager module. The contents added, removed or updated by the provider are managed by the content manager module and stored in a content database. Each content contains an associated model (content model). The provider, aided by the automatic content categorization process, acts in the definition of this model. From the content model, the 
spatial position that the content will occupy in the environment is defined. Content, consumer and provider models are used in the environment adaptation. The representation of the contents in the environment is made by 3D objects and links to the data (e.g., text document, web page). The environment generator module is responsible for the generation of different $3 \mathrm{D}$ structures that form the environment and to arrange the information in the environment, according to user and content models. The environment adaptation involves its reorganization, in relation to the arrangement of the contents and aspects of its layout (e.g., use of different textures and colors, according to user's preferences). Besides, this module transmits to the agent the applicable information about the user interacting with the environment, and information about the contents and their locations, so that it holds enough information in order to assist the users. In the following sections are detailed the main components of the environment.

\subsection{USER MODEL MANAGER}

This module is responsible for initialization and updating of user models. The user model contains information about the users' interests, preferences and behaviors. In order to collect the data used in the composition of the models, the explicit and implicit approaches are used. The explicit approach is adopted to acquire the user's preferences compounding an initial user model and the implicit one is applied to update this model. In the explicit approach, a form is used, to collect the following data: name, e-mail, gender, areas of interest and preferences for colors. The three last data are used in the initial environment adaptation. In the implicit approach, the monitoring of user navigation in the environment and his interactions with the agent are made. Through this approach, the environment places visited by the user and the requested (through the search mechanism) and accessed (clicked) contents are monitored. These data are used to update the initial user model. The process of updating the user model is based on certainty factors (CF) ([Nikolopoulos, 1997]; [Giarratano and Riley, 1998]), which associate measures of belief (MB) and disbelief (MD) in a hypothesis $(\mathrm{H})$, given an evidence $(\mathrm{E}) . \mathrm{A} \mathrm{CF}=1$ indicates total belief in a hypothesis, while a $\mathrm{CF}=-1$ correspond the total disbelief. The calculation of the $\mathrm{CF}$ is accomplished by the following formulas: 


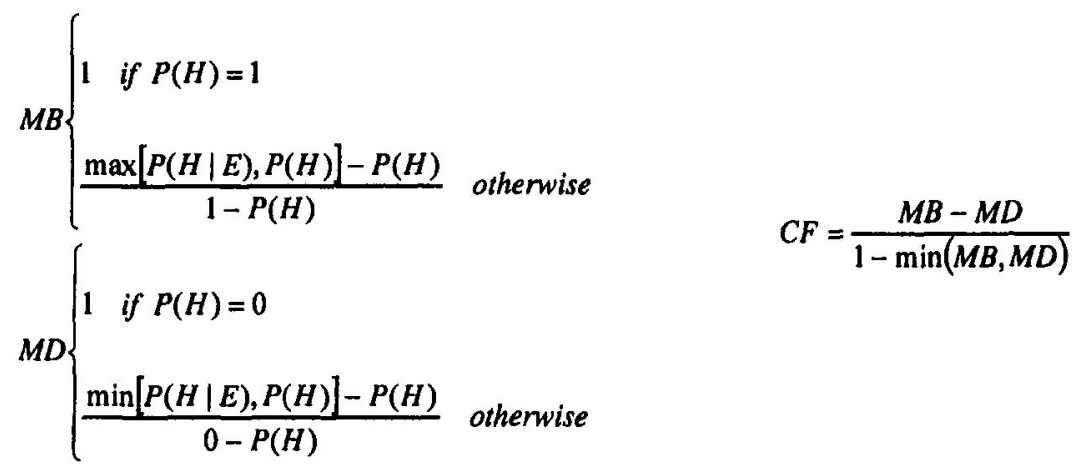

The evidences are related to the environment areas visited and to the requested and accessed contents by the user. They are used to infer the hypothesis of the user's interest in each area of knowledge, from the CF. The user's initial interest in a given area (initial value of $\mathrm{P}(\mathrm{H})$ ) is determined by the explicit data collection and it may vary during the process of updating the model (based on threshold of increasing and decreasing belief). Each $n$ sessions (adjustable time window), for each area, the evidences (navigation, request and access) are verified and the CFs corresponding to the hypothesis of interest are updated. By sorting the resulting CFs, it is possible to establish a ranking of the user's interest area. Therefore it is possible to verify the alterations in the initial model (obtained from the explicit collection) and, thus, to update the user model. From this update, the reorganization of the environment is made - contents that correspond to the areas of major user's interest are placed, in a visualization order, before the contents which are less interesting (easier access). It must be addressed that each modification in the environment is always suggested to user and made only under user's acceptance.

\subsection{CONTENT MANAGER}

This module is responsible for insertion and removal of contents, and management of its models. The provider is responsible for defining the content model, being able to be assisted by an automatic content categorization process. In the insertion of content, the provider must inform the following data, used in the composition of the content model: category (among a pre-defined set), title, description, keywords, type of media and corresponding file. For textual contents, an automatic text categorization process is available, thus the category and the keywords of the content are 
obtained. For non textual contents (for instance, images and videos), textual descriptions of contents can be used in the automatic categorization process.

The categorization process proposed in this work is based on the use of machine learning techniques (see [Sebastiani, 2002]), such as Decision Trees [Quinlan, 1993] and Artificial Neural Networks [Haykin, 2001]. This process is formed by a sequence of stages: (a) document base collection; (b) pre-processing; and (c) categorization. The document base collection consists of obtaining the examples to be used for the training and the test of the learning algorithm. The pre-processing involves, for each example, the elimination of irrelevant words (e.g., articles, prepositions and pronouns), the removal of affix of the words and the selection of the most important words, used to characterize the document. In the categorization stage, the learning technique is then determined, the examples are coded and the classifier learning is accomplished. After these stages, the classifier can be used in the categorization of new documents. In a set of preliminary experiments (details in [Santos and Osorio, 2003]), the decision trees showed to be more robust and were selected for use in the categorization process proposed. In these experiments, the pre-processing stage was supported by an application5, extended from a framelet, whose kernel contemplates the basic flow of data among the activities of removal of irrelevant words and affixes, selection of important words and generation of scripts submitted to the learning algorithms. After applying the learning algorithm, the "learned model" - rules extracted from the decision tree - is connected to the module content manager, in order to use it in the categorization of new documents. Thus, when a new document is inserted in the environment, it is pre-processed, has its keywords extracted and is categorized automatically. Based on the content model, the spatial position where the content must be placed is determined.

\subsection{INTELLIGENT VIRTUAL AGENT}

In the environment, a virtual agent assists users during navigation and retrieval of relevant information. It has the following characteristics: perception of the environment, ability to interact, user/content knowledge, certain degree of reasoning and autonomy and graphic representation. The agent's architecture reflects the following modules: knowledge base, perception, decision and action. The agent's knowledge base stores the information that it holds about the user and the environment. This knowledge is built from two sources of information: external source and

\footnotetext{
${ }^{5}$ Framelet available in: http://www.inf.unisinos.br/ cassiats/dissertacao
} 
perception of the interaction with the user. The external source is the information about the environment and the user, and they are originated from the environment generator module. A perception module observes the interaction with the user, and the information obtained from this observation is used to update the agent's knowledge. It is through the perception module that the agent detects the requests from user and observes the user's actions in the environment. Based on its perception and in the knowledge that it holds, the agent decides how to act in the environment. A decision module is responsible for this activity. The decisions are passed to an action module, responsible to execute the actions (e.g., animation of graphic representation and speech synthesis).

The communication between the agent and the users is made in a verbal way, through a pseudo-natural language and speech synthesis, and non verbal way, by the agent's actions in the environment. The dialogue in pseudo-natural language consists of a certain group of questions and answers and short sentences, formed by a verb that corresponds to the type of user request and a complement, regarding the object of user interest. During the request for helping to locate information, for instance, the user can indicate (in textual interface) Locate <content $>$. The agent's answers are suggested by its own movement through the environment (moving towards the information requested by the user), by indications through short sentences, and by text-to-speech synthesis. In the interaction with provider, during the insertion of content, he can indicate Insert <content>, and the agent presents the data entry interface for the specification, identification and automatic categorization of the content. Moreover, a topological map of the environment is kept in the agent's knowledge base. In this map, a set of routes for key-positions of the environment is stored. In accordance with the information that the agent has about the environment and with the topological map, it defines a set of routes that must be used in the localization of determined content or used to navigate until determined environment area. Considering that the agent updates its knowledge for each modification in the environment, it is always able to verify the set of routes that leads to a new position of a specific content.

\section{PROTOTYPE}

In the prototype, a division of the virtual environment is adopted according to the areas of the knowledge of the contents. In each area a set of sub-areas can be associated. The sub-areas are represented as subdivisions of the environment. In the prototype, the following areas and sub-areas were selected: Artificial Intelligence (AI) - Artificial Neural Networks, Genetic 
Algorithms and Multi Agents Systems; Computer Graphics (CG) Modeling, Animation and Visualization; Computer Networks (CN) Security, Management and Protocols; Software Engineering (SE) - Analysis, Patterns and Software Quality. A room is associated to each area in the environment and the sub-areas are represented as subdivisions of rooms. Figures 1 (a) and 1 (b) show screen-shots of the prototype that illustrate the division of the environment in rooms and sub-rooms. In screen-shots, a system version in Portuguese is presented, where the description "Inteligência Artificial" corresponds to "Artificial Intelligence".

According to the user model, the reorganization of this environment is made: the rooms that correspond to the areas of major user's interest are placed, in a visualization order, before the rooms which contents are less interesting. The initial user model, based on explicit data collection, is used to initial organization of the environment. Figures 2 (a) and 2 (b) represent examples of the initial environment adaptations. In the environment of Figure 2 (a), the user (female gender) has interest by AI and use of clean color; in the environment of Figure 2 (b), the user (male gender) has interest by $\mathrm{CG}$ and use of dark color. As the user interacts with the environment, his model is updated

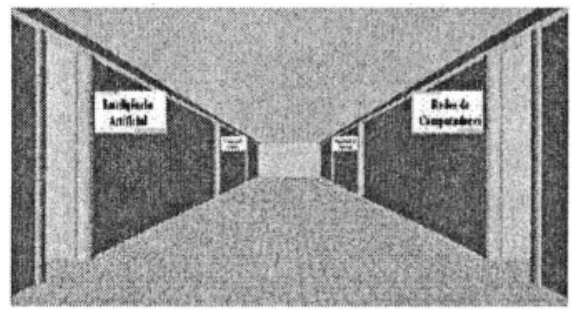

(a)

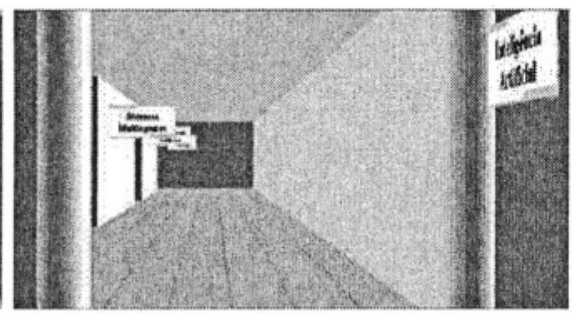

(b)

Figure 1. (a) Rooms and (b) sub-rooms of the environment

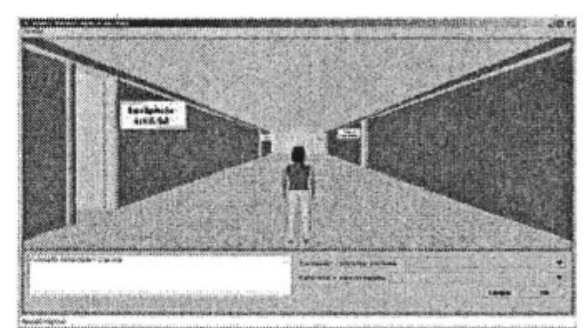

(a)

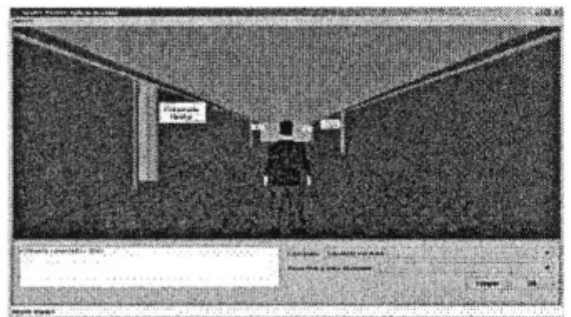

(b)

Figure 2. (a) User with interest in AI; (b) user with interest in CG 


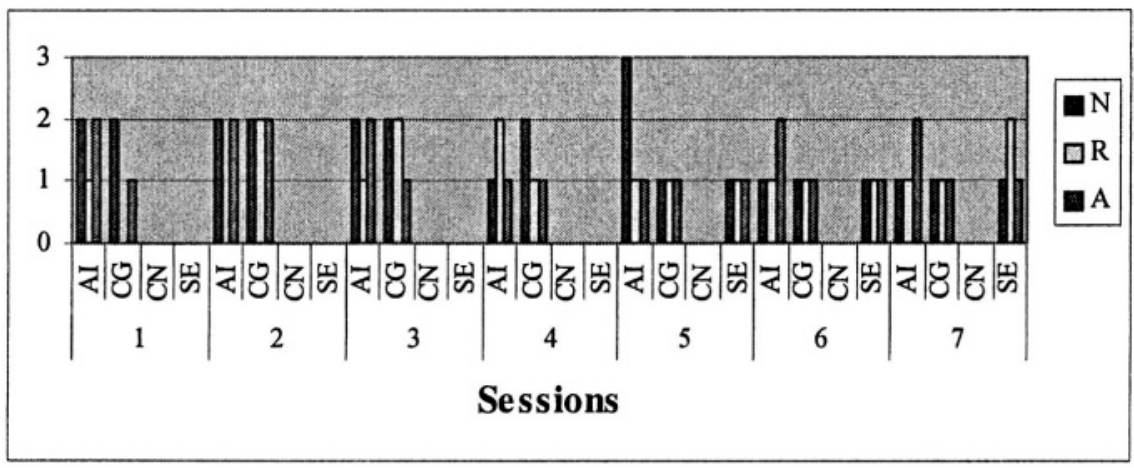

Figure 3. Navigations (N), requests(R) and access (A) for session

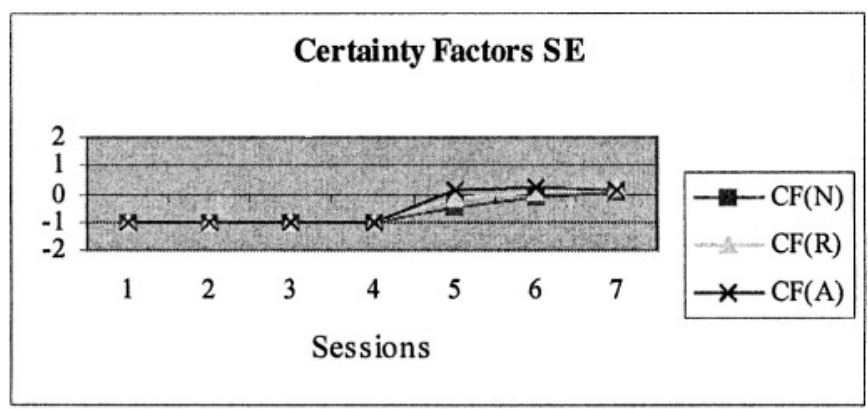

Figure 4. Certainty factors corresponding to evidences of the SE area

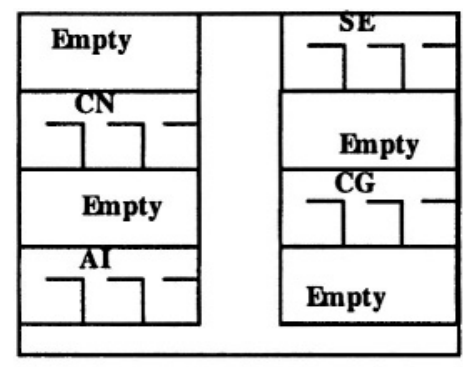

(a)

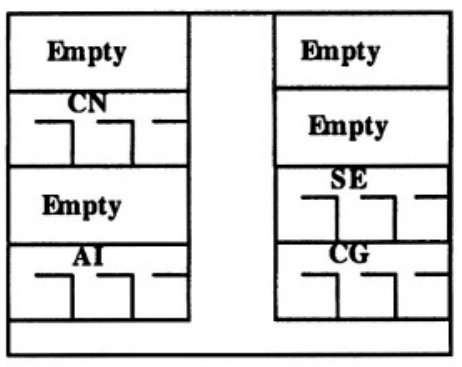

(b)

Figure 5. (a) Organization of the environment according initial user model; (b) organization after the user model changes 
After $\mathrm{n}$ sessions, for each area, the evidences of interest are verified, in order to update the user model. For instance, with a user, who is interested about $\mathrm{AI}$, is indifferent to contents related to the areas of $\mathrm{CN}$ and $\mathrm{CG}$, and does not show initial interest about SE, the initial values of the CFs, at the beginning of the first session of interaction (without evidences), would be respectively $1,0,0 \mathrm{e}-1$. After doing some navigations $(\mathrm{N})$, requests $(\mathrm{R})$ and access (A), presented in the graph of Figure 3, the CFs can be reevaluated. According to Figure 3, it is verified that the $\mathrm{CN}$ area was not navigated, requested and accessed, and on the other side, the user started to navigate, to request, and to access contents in SE area. As presented in the graph of Figure 4, an increasing of the CFs had been identified as related to the SE area. In that way, at end of the seventh session, the resulting CFs would be 1 , $-1,0.4$ and 0.2 (AI, CN, CG, SE, respectively). By sorting the resulting CFs, it would be possible to detect an alteration in the user model, whose new ranking of the interest areas would be AI, CG, SE and CN. Figures 5 (a) and 5 (b) represent an example of the organization of the environment before and after a modification in the user model, as showed in the example above.

On the other side, in relation to manipulation of contents in the environment, the provider model is used to indicate the area (e.g., Artificial Intelligence) that belongs the content being inserted, and the automatic categorization process indicates the corresponding sub-area (e.g., Artificial Neural Nets), or either, the sub-room where the content should be inserted. In this way, the spatial disposal of the content is made automatically by the environment generator, on the basis of its category. In the prototype, thirty examples of scientific papers, for each sub-area, had been collected from the Web, and used for learning and validation of the categorization algorithm. In the stage of learning, experiments with binary and multiple categorizations had been carried through. In the binary categorization, a tree is used to indicate if a document belongs or not to the definitive category. In the multiple categorization, a tree is used to indicate the most likely category of one document, amongst a possible set. In these experiments, the binary categorization presents better results (less error and, consequently, greater recall and precision), being adopted in the prototype. In this way, for each sub-area, the rules obtained from decision tree (C4.5) were converted to rules of type IF - THEN and associated to content manager module.

In relation to communication process between the agent and the users, they interact by a dialog in pseudo-natural language (section 2.3). As a way to simplify the communication model, the user selects one request to the agent in an options list. The agent's answers are showed in the corresponding interface text window and synthesized to speech. Figures 6(a), (b), (c) and (d) illustrate, respectively: a request of the user for the localization of determined area and the movement of agent; the localization of an area by 
the agent; the user visualization of a content; and the visualization of details of a content, after selection and click in a specific content description.

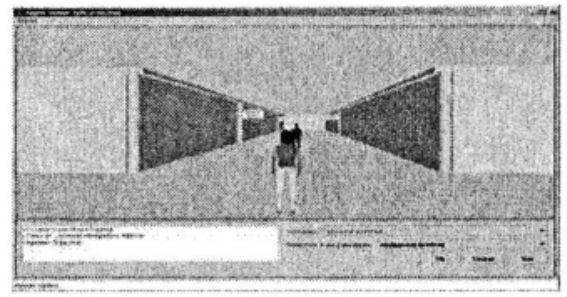

(a)

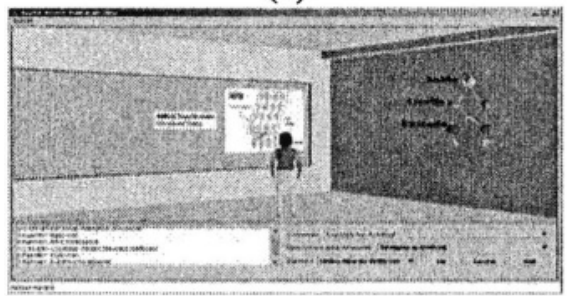

(c)

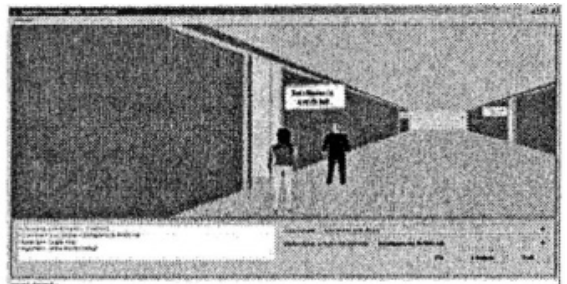

(b)

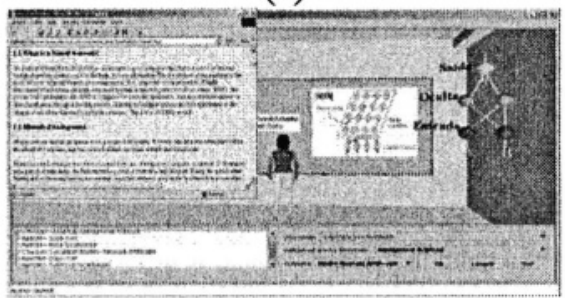

(d)

Figure 6. (a) Request of the user; (b) localization of an area by the agent; (c) visualization of contents; (d) details of a content

\section{FINAL REMARKS}

A large number of distance learning environments make content available through $2 \mathrm{D}$ environments, offering poor interaction with the user. This paper presented an intelligent and adaptive virtual environment that explores the resources of VR, seeking to increase the interactivity degree between the users and the environment. The spatial reorganization possibilities and the environment customization, according to the modifications (addition, removal and updating) in the contents available and the user models were presented. Besides, an automatic categorization process that aims to help the specialist of the domain (provider) in the information organization in this environment was shown. Finally, an intelligent agent that knows the environment and the user and acts assisting him in the navigation and location of information in this environment was commented. An important aspect considered in this work deals with the acquisition of users' characteristics in a 3D environment. Most of the works related to user models acquisition and environment adaptation are accomplished using 2D interfaces. Moreover, a great portion of efforts in the 
construction of Intelligent Virtual Environments don't provide the combination of user models, assisted navigation and retrieval of information, and, mainly, don't have the capability to reorganize the environment, and display the contents in a 3D. Usually, only a sub-group of these problems is considered.

\section{REFERENCES}

Anastassakis, G.; Ritching, T.; Panayiotopoulos, T. 2001. Multi-agent Systems as Intelligent Virtual Environments. LNAI 2174, pp. 381-395.

Avradinis, N.; Vosinakis, S.; Panayiotopoulos, T. 2000. Using Virtual Reality Techniques for the Simulation of Physics Experiments. 4th Systemic, Cybernetic and Informatics International Conference, USA, July.

Aylett, R. and Luck, M. 2000. Applying Artificial Intelligence to Virtual Reality: Intelligent Virtual Environments. Applied Artificial Intelligence, 14(1), pp. 3-32.

Chittaro, L. and Ranon, R. 2002. New Directions for the Design of Virtual Reality Interfaces to E-Commerce Sites. 5th International Conference on Advanced Visual Interface, Italy, May.

Frery, A.; Kelner, J.; Moreira, J., Teichrieb, V. 2002. Satisfaction through Empathy and Orientation in 3D Worlds. CyberPsychology and Behavior, 5(5), pp. 451-459.

Giarratano, J. and Riley, G. 1998. Expert Systems: Principles and Programming. 3 ed., PWS, Boston.

Haykin, S. 2001. Redes Neurais: Princípios e Prática, 2 ed. Bookman.

Nikolopoulos, C. 1997. Expert Systems - Introduction to First and Second Generation and Hybrid Knowledge Based Systems. Eds.: Marcel Dekker, New York.

Quinlan, R. 1993. C4.5: Programs for Machine Learning. Mogan Kaufmann, California,

Rickel, J.; Marsella, S.; Gratch, J.; Hill, R.; Traum, D.; Swartout W. 2002. Toward a New Generation of Virtual Humans for Interactive Experiences. IEEE Intelligent Systems, 17(4).

Santos, C. and Osorio, F. 2003. Uso de Técnicas de Aprendizado de Máquina no Processo de Categorização de Textos. Research (http://www.inf.unisinos.br/ cassiats/mestrado).

Sebastiani, F. 2002. Machine learning in automated text categorization. Computing Surveys, 34(1), pp.1-47.

Teichrieb, V. 1999. Avatares como Guias Interativos para Auxílio na Navegação em Ambientes Virtuais Tridimensionais. Universidade de Pernambuco, Recife, Brazil (Master dissertation). 